\title{
The amazing capacity to read intentions from movement kinematics
}

\section{Sukhvinder S. Obhi*}

Department of Psychology, Centre for Cognitive Neuroscience, Wilfrid Laurier University, Waterloo, ON, Canada *Correspondence: sobhi@wlu.ca

\section{A commentary on}

Grasping intentions: from thought experiments to empirical evidence

by Becchio, C., Manera, V., Sartori, L., Cavallo, A., and Castiello, U. (2012). Front. Hum. Neurosci. 6:117. doi: 10.3389/ fnhum.2012.00117

Becchio et al. (2012) provide an overview of several fascinating and ingenious experiments in which observers are able to discriminate between differing intentions purely based on the kinematics of the observed movement. The range of work presented and the diverse methods that all converge on the same idea is truly impressive and the paper is a very useful contribution to the field. However, although the evidence is clear that "intention reading" of sorts is possible, the scope of this ability remains to be fully explored and the applied implications of this capacity of the human brain require elaboration.

Becchio et al. (2012) frame their paper with respect to a thought experiment proposed by Jacob and Jeannerod (2005). In their thought experiment, Jacob and Jeannerod pose the question of whether an observer could differentiate between the socially helpful intent of Dr. Jekyll when he reaches for a scalpel to operate on an anesthetized patient, and the criminal intent of Mr. Hyde when he reaches for a scalpel to perform the same operation on an unanesthetized patient. Initially, Jacob and Jeannerod suggested that action understanding on the basis of action observation alone could not allow this differentiation, as action understanding relates to the "what" of an action (i.e., the reaching), as opposed to the "why" of an action (i.e., to help someone or hurt someone). So, do the experiments reported in Becchio et al.'s (2012) excellent paper shed light on this thought experiment? Undoubtedly yes, to an extent. In my view however, even in the light of the reported experiments, the answer to the Jekyll and Hyde question is still negative: kinematics alone cannot fully specify the intention of the actor.
The reason for this is that, even in some of the most elegant studies performed, for example by Sartori et al. (2011) and Manera et al. (2011) in which observers were able to discriminate between cooperative, competitive, and individual intentions based on observation of kinematics alone, the choice set of possible intentions was experimentally constrained. If the task for participants was to identify the intention without other boundary conditions, what would the results look like? It seems to me that this type of unconstrained, or less constrained experiment is warranted to more thoroughly test the full scope of the "intention-from-kinematics" ability of the human brain. This comment is in no way to question the importance of the results reported in Becchio et al.'s (2012) article, but simply to point out a limitation in how far we can realistically take this idea, given the current data. So, going back to the Jekyll and Hyde thought experiment, it may be possible for an observer to say that the intent between the two situations differs, but without additional context or a highly constrained choice set, it may be impossible for them to elaborate on exactly what the differences are.

Another very interesting study reported in the paper is Becchio et al. (2008) in which kinematics of a participant's movement were found to reverse on the basis of congruent or incongruent actions of a partner. The logical next step in understanding the full social relevance of this effect is to manipulate the identity of the partner. Is there a partner whose actions cause faster or more definite reversals? Conversely, is there a partner whose actions do not prompt reversals? If such results are found, perhaps a possible application of this technique is as an implicit measure of the degree to which an individual identifies with another person, who may or may not be from a different social group.

Finally, given that competition and cooperation seem to be distinguishable via observation of kinematics, there is tremendous applied scope for the work reported by Becchio et al. (2012). For example, algorithms could potentially be developed to classify kinematics as competitive or cooperative in contexts where these two behavior types are relevant (e.g., military bases, police stations, airports, or even nightclubs and bars). Based on the output of such classifiers, an individual could be "flagged" as a potential threat, and security personnel could be on guard to respond. This form of classification, although perhaps not viable right now, would solve many social problems relating to issues such as racial profiling. With such a system, the term would be "kinematic profiling" and the kinematics may well be irrespective of race or social class.

In sum, the human ability to read intentions from kinematics is fascinating and could explain numerous aspects of our social behavior. Much future work is required to advance our understanding of the capacity and scope of this amazing ability, and I am sure that this work will yield even deeper insights into the social brain.

\section{REFERENCES}

Becchio, C., Manera, V., Sartori, L., Cavallo, A., and Castiello, U. (2012). Grasping intentions: from thought experiments to empirical evidence. Front. Hum. Neurosci. 6:117. doi: 10.3389/fnhum.2012.00117 Becchio, C., Sartori, L., Bulgheroni, M., and, Castiello, U. (2008). Both your intention and mine are reflected in the kinematics of my reach to grasp movement. Cognition 106, 894-912.

Jacob, P., and Jeannerod, M. (2005). The motor theory of social cognition: a critique. Trends Cogn. Sci. (Regul. Ed.) 9, 21-25

Manera, V., Becchio, C., Cavallo, A., Sartori, L., and Castiello, U. (2011). Cooperation or competition? Discriminating between social intentions by observing prehensile movements. Exp. Brain Res. 211, 547-556.

Sartori, L., Becchio, C., and Castiello, U. (2011). Cues to intention: the role of movement information. Cognition 119, 242-252.

Received: 26 April 2012; accepted: 21 May 2012; published online: 08 June 2012.

Citation: Obhi SS (2012) The amazing capacity to read intentions from movement kinematics. Front. Hum. Neurosci. 6:162. doi: 10.3389/fnhum.2012.00162 Copyright $(\odot 2012$ Obhi. This is an open-access article distributed under the terms of the Creative Commons Attribution Non Commercial License, which permits non-commercial use, distribution, and reproduction in other forums, provided the original authors and source are credited. 